\title{
An explicit scaling law for the speed of falling dominoes
}

\author{
Bo-Hua SUN ${ }^{1}$ \\ ${ }^{1}$ Institute of Mechanics and Technology 83 School of Civil Engineering, \\ Xi'an University of Architecture and Technology, Xi'an 710055, China \\ http://imt.xauat.edu.cn \\ email: sunbohua@xauat.edu.cn
}

(Dated: December 4, 2019)

\begin{abstract}
By using directed dimensional analysis and data fitting, an explicit universal scaling law for the speed of falling dominoes is formulated. The scaling law shows that domino propagational speed is linear proportional to the $3 / 2$ power of domino separation, and $-1 / 2$ power of domino height and thickness.
\end{abstract}

Keywords: domino, speed, height, thickness, separation

\section{INTRODUCTION}

The falling of dominoes is a successive toppling of regularly spaced elements in a periodic array plotted in Figure 1. The domino effect is not only an interesting game but also an important physical phenomena, and often be used to describes some social catastrophe, such as the cascading consequences of research misconduct [1].

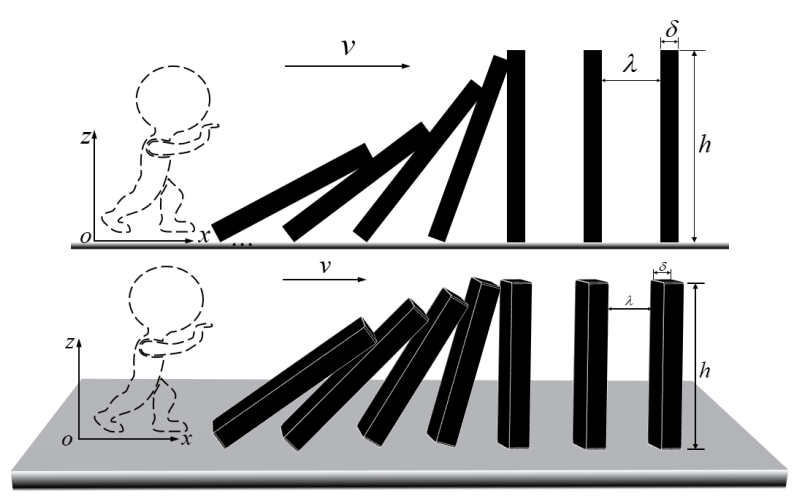

FIG. 1: The falling dominoes.

The mechanics of domino falling has been studied extensively by number of leading scholars [2-14]. In 1983, McLachlan et al. [4] found a scaling law for the speed $v$ in the limiting case of dominoes with zero thickness spaced in a straight line. With these assumptions McLachlan et al. [4] the functional relation:

$$
v_{\text {McLachlan }}=v(h, \lambda, g)=\sqrt{g h} f\left(\frac{\lambda}{h}\right)
$$

here $g$ is gravitation acceleration, $h$ the height of the dominoes, $\lambda$ the spacing between dominoes, and $f(x)$ an undetermined function of $x$. Efthimiou and Johnson [6] proposed a $f(x)$ by complete elliptic integral of the first kind. Shi et al. [13] developed a precise numerical model with consideration of multipoint impacts between dominoes. Shi et al. [14] studies the toppling dynamics of a mass-varying domino system for which the mass of the domino changes at an exponential rate of its sequence number.

Szirtes and Rozsa [6] studied domino by using dimensional analysis [15-19] for a domino with equal thickness $\delta$, separation $\lambda$ and height $h$. Hence the five variables and their diemsion are listed in Table I below:

TABLE I: Dimensions of physical quantity

\begin{tabular}{c|c|c}
\hline Variables & Symbol & Dimension \\
\hline \hline velocity & $v$ & $\mathrm{LT}^{-1}$ \\
\hline height & $h$ & $\mathrm{~L}$ \\
\hline thickness & $\delta$ & $\mathrm{L}$ \\
\hline separation & $\lambda$ & $\mathrm{L}$ \\
\hline gravitational acceleration & $g$ & $\mathrm{LT}^{-2}$ \\
\hline
\end{tabular}

The dimensional basis used is length $(\mathrm{L})$ and time $(\mathrm{T})$.

Szirtes and Rozsa [6] applied dimensional analysis to find domino velocity, $v=v(h, \lambda, \delta, g)$. The problem has five variables and two dimensions $(\mathrm{L}, \mathrm{T})$, therefore there are $5-2=3$ dimensionless variables $\Pi$ as follows:

$$
\Pi_{1}=\frac{v}{\sqrt{g h}}, \quad \Pi_{2}=\frac{\lambda}{h}, \quad \Pi_{3}=\frac{\delta}{h},
$$

From dimensional analysis, $\Pi_{1}=f\left(\Pi_{2}, \Pi_{3}\right)$, namely

$$
v_{\text {Szirtes }}=\sqrt{g h} f\left(\frac{\lambda}{h}, \frac{\delta}{h}\right) .
$$

This relation is similar to Eq.(1) except the separation and height ratio $\delta / h$.

Although we have Eq.(1) and Eq.(3), there is no much useful information can be get from them, because the function $f\left(\frac{\lambda}{h}, \frac{\delta}{h}\right)$ is still undetermined. In the following, we will try to decode the function by using directed dimensional analysis proposed by Huntley [20] and Siano $[21,22]$.

\section{DIRECTED DIMENSIONAL ANALYSIS I}

According to the directed dimensional analysis, we can distinct the length dimension in both $x$ and $z$ direc- 
tion. The problem has five variables and three dimensions $\left(\mathrm{L}_{x}, \mathrm{~L}_{z}\right.$ and $\left.\mathrm{T}\right)$ listed in Table IV below:

TABLE II: Dimensions of physical quantity

\begin{tabular}{c|c|c}
\hline Variables & Symbol & Dimension \\
\hline \hline velocity & $v$ & $\mathrm{~L}_{\mathrm{x}} \mathrm{T}^{-1}$ \\
\hline height & $h$ & $\mathrm{~L}_{\mathrm{z}}$ \\
\hline thickness & $\delta$ & $\mathrm{L}_{\mathrm{x}}$ \\
\hline separation & $\lambda$ & $\mathrm{L}_{\mathrm{x}}$ \\
\hline gravitational acceleration & $g$ & $\mathrm{~L}_{\mathrm{z}} \mathrm{T}^{-2}$ \\
\hline
\end{tabular}

The dimensional basis used is length $\left(\mathrm{L}_{x}, \mathrm{~L}_{z}\right)$ and time $(\mathrm{T})$.

Therefore there are $5-3=2$ dimensionless variables $\Pi$ as follows:

$$
\Pi_{1}=v h^{a} \lambda^{b} g^{c}, \quad \Pi_{2}=\delta h^{a_{1}} \lambda^{b_{1}} g^{c_{1}},
$$

where the exponents $a, b, c$ and $a_{1}, b_{1}, c_{1}$ can be determined by following dimensionless conditions: $\operatorname{dim}\left(\Pi_{1}\right)=$ $\operatorname{dim}\left(\Pi_{2}\right)=L_{x}^{0} L_{z}^{0} T^{0}$, namely

$$
\begin{aligned}
\operatorname{dim}\left(\Pi_{1}\right) & =L_{x} T^{-1}\left(L_{z}\right)^{a}\left(L_{x}\right)^{b}\left(L_{z} T^{-2}\right)^{c} \\
& =L_{x}^{1+b} T^{-1-2 c} L_{z}^{a+c} .
\end{aligned}
$$

From dimensionless condition, $1+b=0,-1-2 c=0$ and $a+c=0$, leads to $a=\frac{1}{2}, b=-1$ and $c=-\frac{1}{2}$. Hence, we have the first dimensionless variable

$$
\Pi_{1}=\frac{v}{\lambda} \sqrt{\frac{h}{g}} .
$$

Similarly, we have $a_{1}=0, b_{1}=-1$ and $c_{1}=0$ and the second dimensionless variable

$$
\Pi_{2}=\frac{\delta}{\lambda}
$$

From Buckingham dimensional theorem [15], the domino speed $v=v(h, \lambda, \delta, g)$ can be replaced by $\Pi_{1}=f\left(\Pi_{2}\right)$ as follows

$$
v=\lambda \sqrt{\frac{g}{h}} f\left(\frac{\delta}{\lambda}\right) .
$$

This relation has not been reported in literature, however, the function $f\left(\frac{\delta}{\lambda}\right)$ is still undetermined.

\section{DIRECTED DIMENSIONAL ANALYSIS II}

In order to reduce the number of variables, let's consider the domino's area instead of its height and thickness separately. The problem has four variables and three dimensions $\left(\mathrm{L}_{x}, \mathrm{~L}_{z}\right.$ and $\left.\mathrm{T}\right)$ listed in Table IV below:

Therefore there are $4-3=1$ dimensionless variable $\Pi$ as follows:

$$
\Pi=v A^{a} \lambda^{b} g^{c}
$$

TABLE III: Dimensions of physical quantity

\begin{tabular}{c|c|c}
\hline Variables & Symbol & Dimension \\
\hline \hline velocity & $v$ & $\mathrm{~L}_{\mathrm{x}} \mathrm{T}^{-1}$ \\
\hline area & $A$ & $\mathrm{~L}_{\mathrm{x}} \mathrm{L}_{\mathrm{z}}$ \\
\hline separation & $\lambda$ & $\mathrm{L}_{\mathrm{x}}$ \\
\hline gravitational acceleration & $g$ & $\mathrm{~L}_{\mathrm{z}} \mathrm{T}^{-2}$ \\
\hline
\end{tabular}

The dimensional basis used is length $\left(\mathrm{L}_{x}, \mathrm{~L}_{z}\right)$ and time $(\mathrm{T})$.

The dimension $\operatorname{dim}(\Pi)=L_{x}^{1+a+b} T^{-1-2 c} L_{z}^{a+c}=$ $L_{x}^{0} T^{0} L_{z}^{0}$, hence, $a=\frac{1}{2}, b=-\frac{3}{2}$ and $c=-\frac{1}{2}$.

Since we have only one dimensionless variable $\Pi$, therefore, this $\Pi$ can only be a constant, namely

$$
v=C \frac{\lambda^{3 / 2}}{\sqrt{g A}}=C \frac{\lambda^{3 / 2}}{\sqrt{g h \delta}},
$$

where the area of rectangular domino $A=h \delta$ is used. The beauty of Eq.(10) is that it is an explicit formula for all relevant variables. The only unknown is the constant $C$, which can be determined by experiment.

This remarkable relation reveals that, for given domino's dimensions $h$ and $\delta$, the domino wave prorogation speed is proportional to the $3 / 2$ power law of domino's separation $\lambda$. The gradient of speed respect to the separation $\lambda$ is $\frac{d v}{d \lambda}=\frac{3}{2} C \sqrt{\frac{\lambda}{g h \delta}}$. Therefore, the domino's separation $\lambda$ is a control and/or dominate parameter for the speed of domino motion.

Stronge [9] conducted comprehensive study with highspeed photography on topping of domino array, who obtained three data for domino dimensions: $h=41.78 \mathrm{~mm}$, $\delta=7.58 \mathrm{~mm}$ :

TABLE IV: Experimental data from Stronge [9]

\begin{tabular}{c|c|c|c}
\hline height & thickness & separation & velocity \\
\hline \hline $\begin{array}{c}h \\
(\mathrm{~m})\end{array}$ & $\begin{array}{c}\delta \\
(\mathrm{m})\end{array}$ & $\begin{array}{c}\lambda \\
(\mathrm{m})\end{array}$ & $\begin{array}{c}v \\
(\mathrm{~m} / \mathrm{s})\end{array}$ \\
\hline 0.04178 & 0.00758 & 0.0219 & 0.65 \\
\hline 0.04178 & 0.00758 & 0.02949 & 0.80 \\
\hline 0.04178 & 0.00758 & 0.03419 & 0.86 \\
\hline
\end{tabular}

Using the data from the above table, we can calculate the constant by $C=v \frac{\sqrt{g h \delta}}{\lambda^{3 / 2}}$, hence, we have $C_{1}=$ 11.17323782, $C_{2}=8.800547398$ and $C_{3}=7.578472140$, and its average $C \approx\left(C_{1}+C_{2}+C_{3}\right) / 3=9.18 \approx 2.923 \pi$. Finally, we have an explicit speed of falling dominoes as follows:

$$
v=2.923 \pi \frac{\lambda^{3 / 2}}{\sqrt{g h \delta}} .
$$

This explicit scaling law for the speed of falling dominoes has never been reported in literature before. 


\section{CONCLUSION}

In conclusion, an explicit universal scaling law for the speed of falling dominoes has been formulated by using directed dimensional analysis. It is surprised to see that the domino speed is not linearly proportional to $\sqrt{g h}$ as reported in literature (McLachlan [4] and Szirtes and Rozsa [6]). The study also shown that the domino wave prorogation speed is proportional to the $3 / 2$ power law of domino's separation $\lambda$. which reveals that the domino's separation $\lambda$ is a control parameter for domino motion.

Acknowledgement: The author appreciates the financial supports from Xi'an University of Architecture and Technology and Mr Zhe Liu for the preparation of Figure 1.

[1] David M. Polaner, MD, FAAP, and Steven L. Shafer, Falling Dominoes, Anesthesia \& Analgesia, 128(4):613614 (2019)

[2] Daykin, D.E.: Falling dominoes. SIAM Review 13(4), 569 (1971)

[3] Shaw, D.E.: Mechanics of a chain of dominoes. Am. J. Phys. 46(6), 640-642 (1978)

[4] McLachlan, B.G., Beaupre, G., Cox, A.B., Gore, L. Falling dominoes (de daykin). SIAM Rev. 25(3), 403 (1983)

[5] Bert, C.W.: Falling dominoes. SIAM Rev. 28(2), $219-224(1986)$

[6] Szirtes, T and Rozsa, P. Applied Dimensional Analysis and Modelling, Elsevier Science \& Technology Books, 2006.

[7] Efthimiou, C.J., Johnson, M.D.: Domino waves. SIAM Rev. 49(1), $111-120$ (2007)
[8] Larham, R.: Validation of a Model of the Domino Effect? arXiv:0803.2898 (2008)

[9] Stronge, W.J.: The domino effect: a wave of destabilizing collisions in a periodic array. Proc. R. Soc. A Math. Phys. Eng. Sci. 409(1836), 199 - 208 (1987)

[10] Stronge,W.J., Shu, D.: The domino effect: successive destabilization by cooperative neighbours. Proc. R. Soc. A Math. Phys. Eng. Sci. 418(1854), 155 - 163 (1988)

[11] VanLeeuwen, J.M.J.:The domino effect.Am. J. Phys. $78(7), 721-727(2010)$

[12] Fujii, F., Inoue, Y., Nitta, T.: Modeling the domino wave propagation in contact mechanics. Trans. Jpn. Soc. Mech. Eng. Ser. C 78(788), 1133 - 1142 (2012)

[13] Shi, T., Liu, Y., Wang, N., Liu, C.: Toppling dynamics of regularly spaced dominoes in an array. J. Appl.Mech. 85(4), 041008 (2018)

[14] Shi, T., Liu, Y., and Wang, N. Toppling dynamic$\mathrm{s}$ of a mass-varying domino system. Nonlinear Dyn. https://doi.org/10.1007/s11071-019-05324-8 (2019)

[15] Bridgman, P.W. Dimensional Analysis. Yale University Press, New Haven (1922).

[16] Sun, B.H. Dimensional Analysis and Lie Group, China High Education Press, Bejing (2016).

[17] Sun, B.H. Scaling laws of compressible turbulence, Appl. Math. Mech. 38: 765(2017).

[18] Sun, B.H. Capillary wrinkling scaling laws of floating elastic thin film with a liquid drop, Sci. China-Phys. Mech. Astron. 61, 024721 (2018).

[19] Sun, B.H. Kepler' s third law of n-body periodic orbits in a Newtonian gravitation field, Sci. China-Phys. Mech. Astron. 61, 054721 (2018).

[20] Huntley, H. E. Dimensional Analysis. Dover,1967.

[21] Siano, D. Orientational Analysis - A Supplement to Dimensional Analysis - I. J. Franklin Institute, 1985, 320:267.

[22] Siano, D. Orientational Analysis - Tensor Analysis and The Group Properties of the SI Supplementary Units - II. J. Franklin Institute, 1985, 320:285. 